\title{
1. A Comparative study to observe the effect of Meditation in Hypertension
}

Rajesh Kolarkar, Professor, YMT Ayurvedic Medical college, Mumbai.

Rajashree Koloarkar, Reader, Smt.K.G.Mittal Ayurved college, Mumbai

\begin{abstract}
Aims and Objectives :
Primary objective was to evaluate and compare the change or reduction in systolic and diastolic blood pressure (BP) in sitting position from baseline (week 0) to the end point (Week 12) between two groups, to evaluate and compare the change or reduction in Stress value between two groups and Secondary Objective was to observe and compare change in lipid profiles between two groups.
\end{abstract}

\section{Methods:}

In a comparative interventional study Group A 30 patients of hypertension of male and female were given training in relaxation and meditation. Study was performed in YMT Ayurvedic Medical College and Smt. K.G. Mittal college and Sanjivani Ayurvedic centre Mumbai.The study was conducted on 30 patients including both male and female practicing daily meditation for 12 weeks to observe effect of meditation in hypertension. This showed an overall reduction of systolic and diastolic blood pressure.

\section{Results:}

In a controlled trial, 30 patients with systemic hypertension were given training in relaxation and meditation. As a result there was a significant reduction in both systolic and diastolic pressure in $75 \%$ of the patients. In $50 \%$ of the patients it was also possible to reduce antihypertensive drugs.

\section{Conclusion -}

the study has shown a significant reduction in both systolic and diastolic pressure

\section{Introduction:}

Meditation is the highest method of mystical practice. Charakacharya has described Yoga is the last and best medicine for all types of misery. In the classic scheme of advancement through the stages spiritual development outlined by Patanjali meditation (Dhyana) is on the seventh place. It is preceded by learning the ethical principles, the rules of hygiene, making the body healthy with the help of special physical exercise, cleansing, learning to control of the mind and many other things.

Vipassana Meditation is the process of self purification by self observation one being by observing the natural breath to concentrate the mind then with a sharpened awareness. One proceeds to observe the changing nature of body and mind and experiences the universal truth of impermanence suffering agelessness. This truth realization by direct experience results in mental purification. Numerous scientific investigations have undoubtedly proved that psychological stress can have disastrous effect on the physical health. In diseases like hypertension, diabetes, acidity, Peptic ulcer, and Bronchial asthma psychological stress is one of the factors for causing or complicating the disease process and also disturbs recovery with pharmacological treatment

\section{Mode of action:}

Psychological stress stimulates the secretion of the hypothalamic factors which in turn derange the secretion of the pituitary hormones. The hormonal changes disorganize the metabolism and the physiology of the organs. This change disturbs our psyche.

\section{Disease:}


Hypertension or high blood pressure is a chronic medical condition in which the systemic arterial blood pressure is elevated.

In the year 2000 it was estimated that nearly one billion or $26 \%$ of the adult population had hypertension worldwide. Persistent hypertension is one of the risk factors for stroke, myocardial infarction, heart failure, and arterial aneurysm and a leading cause of chronic kidney failure. Patient has to spend lot of money on the treatment of hypertension.

Current method of staging blood pressure

Systolic Diastolic

Normal 120 or $<120<80$

Pre hypertension 120 - 14080 to 90

Stage - I Hypertension 140 - 16090 to 100

Stage - II Hypertension >160>100

Only when the disease is advanced some persons displays signs and symptoms of high blood pressure Such as headache, blurred vision, nausea and dizziness. Most of the people do not seek medical care until they have more severe symptoms from the organ damage that long term (chronic) high blood pressure can cause.

\section{Subjects and methods:}

The goal of treatment is to reduce blood pressure so that you have a lower risk of complication and to save the expense on medicine.

High blood pressure can be controlled with diet, medical and life style changes. There are following main classes of drugs used for controlling blood pressure Diuretics, Beta blocker, Ca channel blocker, ACE inhibiters etc.

Despite all the advances in hypertension diagnosis and therapy there are many patients who develop complex side effect and also remain uncontrolled. Above no one class is unable to control hypertension and treatment is very expensive.

\section{Primary objective:}

To evaluate and compare the change or reduction in systolic and diastolic blood pressure(BP) in sitting position from baseline (week 0 ) to the end point (WEEK 12) between two groups.

To evaluate and compare the change or reduction in Stress value between two groups.

\section{Secondary Objective:}

To observe and compare change in lipid profiles between two groups.

\section{Treatment:}

Eligible patients were given daily one hour meditation for 12 weeks and explained good conducts.

\section{Study population:}

Total of minimum 60 patients were enrolled in the study and randomized such that there were 30 patients in each group. To achieve this goal we were enrolled 80 patients. Among 80 patients 10 patients dropped out from the study

\section{Inclusion criteria:}

All the patients will be selected and followed up by cardiologist in the study

1) Male or non pregnant female.

2) Age - 30to70 years

3) Patients on treatment and are not controlled

4) Written informed consent and patients willing to follow up.

\section{Exclusion criteria:}

- Pregnancy and lactation

- Patient with severe hypertension, malignant hypertension and secondary hypertension.

- Patient with a history of MI, unstable angina or arrhythmias.

- Patient of CCF.

- Patient with stroke.

- Mentally challenged cases.

- Patient with significant, cardiac, hepatic, renal, cerebrovascular diseases, Diabetic mellitus and malignancy.

- Uncontrolled endocrine or metabolic diseases, hepatic failure. 
- Impaired renal function.

- Any condition which is likely to hamper compliance of the patient during the study.

\section{Study assessment:}

\section{Primary Parameter:}

To evaluate and compare the change or reduction in systolic and diastolic blood pressure(BP) in sitting position from baseline (week 0) to the end point (WEEK 12) between two groups.

To evaluate and compare the change or reduction in Stress value between two groups.

\section{Secondary Parameter:}

To observe and compare change in lipid profiles between two groups

Over all global assessment of efficacy by the physician was done from second visit onwards and also patient was carried out at all the visit following grades.

- Excellent - Complete resolution of signs and symptoms

- Good - Moderate resolution of signs and symptoms (Moderate improvement)

- Fair - Small improvement in sign and symptom (Mild improvement)

- Poor - disease unchanged or deteriorated.

\section{Physical Examination:}

- A physical examinations were performed at the base line visit as follows- Temperature, Respiration rate, Pulse rate, CVS,CNS,RS,Skin,Musculoskeletal system,endocrine,ENt and eyes.

- Laboratory investigations was performed at baseline and end of treatment as fallows CBC,Urine,lipid profile.

- ECG was done to determine the changes before and after treatment in both the groups.

\section{Study Treatment}

1) Grop A - was treated by Rx of HTN with meditation

2) Grop A - was treated by only Rx of HTN

Study was performed in YMT Ayurvedic Medical College and Smt.K.G. Mittal college and Sanjivani Ayurvedic centre Mumbai.

\section{STATISTICAL ANALYSIS:}

- Qualitative (Text) data will be presented in form of frequency (count) and percentage.

- Quantitative (numerical) data will be presented in form of mean \pm standard deviation and median, IQR (IQR= Interquartile Range (i.e. 75th Percentile-25th Percentile)).

- Analysis of Qualitative (Text) data between the 2 groups will be done using Chi-Square test with Continuity Correction for all 2 row X 2 columns tables. Where Chi-Square test is invalid due to expected count is less than 5 in more than $20 \%$ table cells, Fisher's Exact Test will be used.

- Analysis of Qualitative (Text) data before and after intervention within each group will be done using McNemar test.

- Analysis of Quantitative (numerical) data of ordinal type (scale, score etc.) between the 2 groups will be done using Mann-Whitney test.

- Analysis of Quantitative (numerical) data of interval type between the 2 groups will be done using unpaired $t$ test if data passes Normality Test or Mann-Whitney test if data fails Normality Test.

- Analysis of Quantitative (numerical) data of ordinal type (scale, score etc.) before and after intervention within each group will be done using Wilcoxon Signed Rank Test.

- Analysis of Quantitative (numerical) data of interval before and after 


\section{Ayurlog: National Journal of Research in Ayurved Science}

A Web based quarterly online published peer reviewed National E-journal of Ayurveda.

intervention within each group will be done using Paired t-test if data passes Normality Test or Wilcoxon Signed Rank Test if data fails Normality Test.

- Data will be represented graphically as deemed necessary.

Results - The study was conducted on 30 patients including both male and female practicing daily meditation for 12 weeks to observe effect of meditation in hypertension. This showed an overall reduction of systolic and diastolic blood pressure.

Further studies are still going on in our institute.

\section{References:}

1. Blood pressure response to transcendental meditation: a meta-analysis. Anderson JW, Liu C, Kryscio RJ.

2. Meditation training and essential hypertension: a methodological study. Seer P, Raeburn JM

3. Relaxation and biofeedback techniques in the management of hypertension. Patel C, Datey KK 